
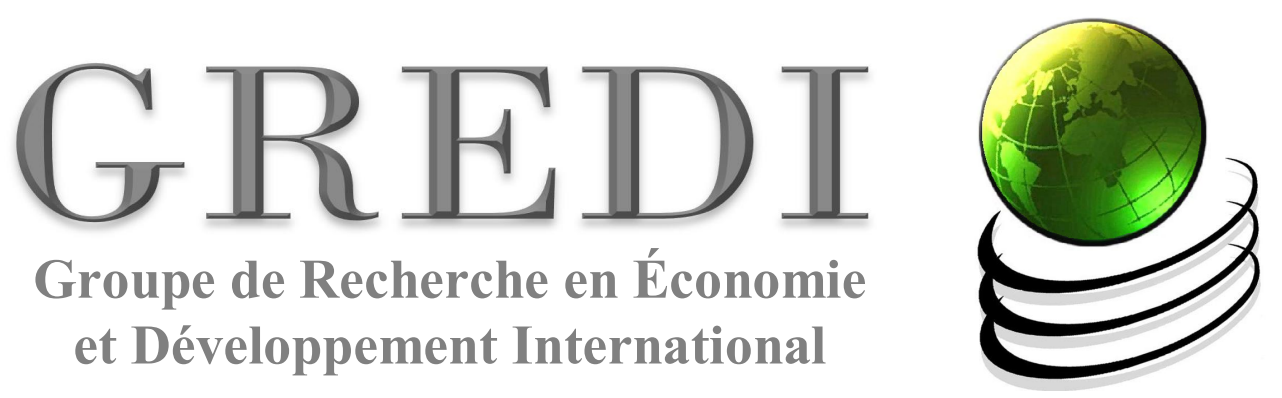

\author{
Cahier de Recherche / Working Paper \\ 10-29
}

A Simple Bargaining Procedure for the Myerson Value

Noemí Navarro

Andrés Perea 


\title{
A Simple Bargaining Procedure for the Myerson Value*
}

\author{
Noemí Navarro Andrés Perea
}

This version: November 2010

\begin{abstract}
We consider situations where the cooperation and negotiation possibilities between pairs of agents are given by an undirected graph. Every connected component of agents has a value, which is the total surplus the agents can generate by working together. We present a simple, sequential, bilateral bargaining procedure, in which at every stage the two agents in a link $(i, j)$ bargain about their share from cooperation in the connected component they are part of. We show that, if the marginal value of a link is increasing in the number of links in the connected component it belongs to, then this procedure yields exactly the Myerson value payoff (Myerson, 1977) for every player.
\end{abstract}

Keywords and phrases: Myerson value, networks, bargaining, cooperation.

JEL Classification: C71, C72.

Noemí Navarro: Université de Sherbrooke, Departement d'Économique et GREDI. E-mail: noemi.navarro@usherbrooke.ca

Andrés Perea (Corresponding author): Maastricht University, Department of Quantitative Economics, P.O. Box 616, 6200 MD Maastricht, The Netherlands.

E-mail: a.perea@maastrichtuniversity.nl

*Part of this work was written while Navarro was visiting CIRPÉE, UQàM. She is grateful for their hospitality. The usual disclaimer applies. 


\section{Introduction}

This paper introduces a noncooperative bargaining procedure that leads to the Myerson value (Myerson, 1977) for network games. The Myerson value has been widely used as an exogenously given payoff scheme in many non-cooperative settings, although the choice of allocation rules, or equivalently, payoff schemes is quite large. Network games range from the seminal work by Myerson (1977), in which cooperative TU games in characteristic function form are restricted to communication structures, to the more general specification introduced by Jackson and Wolinksy (1996), in which the value function takes the network as a primitive. (See the book by Jackson (2008), Chapter 12, for a more formal definition). Apart from the Myerson value, many other allocation rules have been proposed and studied for network games. See, for example, Amer and Carreras (1995), Bergantiños, Carreras and García-Jurado (1993), Borm, Owen and Tijs (1992), Calvo, Lasaga and van den Nouweland (1999), Hamiache (1999), Herings, van der Laan and Talman (2008), Jackson (2005), Slikker (2005), Slikker, Gilles, Norde and Tijs (2005), Talman and Yamamoto (2008) and Vázquez-Brage, García-Jurado and Carreras (1996).

The Myerson value is relatively easy to compute. Nevertheless, that is not the only reason for its popularity. The Myerson value presents appealing properties when applied to network formation settings. ${ }^{1}$ Namely, it is well-known that some non-cooperative games of network formation have a potential when the payoffs for players follow the Myerson value in each of the possible resulting networks. ${ }^{2}$ A similar result is known about the Shapley value and noncooperative games of coalition formation. ${ }^{3}$ In addition, Jackson (2003) shows that there always exists a pairwise stable network relative to the Myerson value and, even more importantly, following improving paths with respect to the Myerson value always leads to a pairwise stable network. These are very important features of the Myerson value, since the existence of pairwise stable networks or them being a "sink" of a dynamic network formation setting is not always granted.

In this paper we propose a simple bargaining procedure in network games that leads to the Myerson value if the surplus from cooperation is link-convex. Our bargaining procedure consists of a sequence of bilateral negotiations between agents. First, we fix a graph. Each link in that graph represents a pair of agents that are allowed to bargain directly. Afterwards, we fix a rule of order over the links of the graph. When a pair of agents is called to play, they bargain in two stages, called "bid" and "propose" stages. During the "bid" stage agents announce a bid price. During the "propose" stage the agent with the highest bid proposes a payoff for him and for the other player. In case of acceptance by the other player, both players commit to the proposed pair of payoffs and the mechanism turns to the next link given by the rule of order. In case of rejection (i) the proposer has to pay his announced bid to the other player, (ii) the two players are not allowed to bargain directly any more, and (iii) the procedure starts all over again. Precisely, the graph that is fixed after the rejection corresponds to the current graph where the link representing the pair of players that could not reach an agreement has been eliminated.

\footnotetext{
${ }^{1}$ See for example Aumann and Myerson (1988), Dutta, van den Nouweland and Tijs (1998), Garrat and Qin (2003a) and (2003b), Pin (2008), Slikker, Dutta, van den Nouweland and Tijs (2000) and Slikker and van den Nouweland (2000, 2002).

${ }^{2}$ For a definition of games with potential, see Monderer and Shapley (1996).

${ }^{3}$ See Qin (1996).
} 
The procedure continues until all the links in the original graph have been considered.

The final result of the procedure will consist of a series of bilateral agreements on payoffs and a graph, possibly empty, which includes all directly connected pairs of agents that have reached an agreement. For every connected component in the final graph, we first check that the total amount of agreed upon payoffs by the participating agents in the component does not exceed the value of the component. Provided this is true, every agent receives the sum of his agreed upon payoffs at the final graph, plus or minus the bids received or paid as a result of previous disagreements. Otherwise, the empty network is implemented and everybody receives his or her stand-alone payoff, plus or minus the bids received or paid as a result of disagreements.

We prove that, under the assumption that the surplus from cooperation is link-convex, there is a unique subgame perfect equilibrium outcome to this procedure in which all proposed payoffs are accepted and the final payoffs for the players coincide with the Myerson value applied to the original graph. Here, by link-convex we mean that the marginal contribution of a link to a connected component is increasing in the number of links within this component. We show that this implies that the Myerson value payoff for all agents in a component increases by adding a link to this component.

The monotonicity of the Myerson value with respect to the density of the network inside a component is a relevant requirement due to two features of our mechanism. First, the bargaining procedure is sequential. And second, in case of disagreement, the link connecting the pair of agents who did not reach an agreement disappears from the bargaining procedure. The sequentiality of the procedure could in principle induce a first-mover advantage: Two agents bargaining now can agree on payoffs that are too high. This, in turn, could leave not enough payoff for some other players that will bargain later during the procedure. In other words, two agents agreeing on payoffs that are too high could lead to a future disagreement. In case of disagreement the corresponding link is eliminated from the graph. Therefore, if link-convexity is satisfied, there is no incentive for disagreement, as the resulting payoffs of the bargaining procedure after a rejection are smaller for everybody. The same type of condition appears in part of the literature implementing the Shapley value. The Demand Commitment Game is a well-known game form that has been proven to lead to the Shapley value by Winter (1994) and by Dasgupta and Chiu (1998). In such a game, agents sequentially commit to join any coalition meeting his payoff demand. The main difference of these two works is the following. In Winter (1994) the first agent is randomly chosen and each agent points at the next player after announcing the demand, while in Dasgupta and Chiu (1998) there is a random order over the whole set of agents. Both require the TU game to be convex, which in turn means that the Shapley value payoff of each agent is increasing any time a new agent, or new set of agents, joins the cooperation. Therefore, no agent has an incentive of demanding too much, forcing some agents out of cooperation from the grand coalition. In a recent working paper, de Fontenay and Gans (2007) scape from the trap of convexity when implementing the Myerson value in a bilateral sequential setting by assuming (i) that only breakdowns, and not agreed-upon payoffs, are common knowledge, and (ii) that agents hold "passive beliefs" about unobservable actions (i.e., the agreed upon payoffs inside the links in which they are not participating). This assumption of passive beliefs implies that agents believe that all unobservable actions take place as in the equilibrium path and, in case of receiving an offer out of equilibrium, they do not update beliefs about the unobservable actions. Therefore, there is no room for foreseeing 
future rejections as a result of a current (possible) out-of-equilibrium agreements, which, as mentioned above, was causing the need of a convexity-type requirement on the value function. De Fontenay and Gans (2007) are then able to implement the Myerson value under passive beliefs for component super-additive value functions. One last comment is in order. Dasgupta and Chiu (1998) define a system of transfers and taxes so that the Demand Commitment Game could implement the Shapley value for any TU game. On one hand, the system of transfers makes larger coalitions more valuable and, on the other hand, the system of taxes alleviates the first-mover advantage (apart from balancing the budget), since the agents coming first by the rule of order pay more than the ones that come after. Whether a system of transfers and taxes could be possible in our setting we leave it for future research.

Other mechanisms leading to the Shapley value are the ones proposed by Hart and MasColell (1996) and the bidding-for-the-surplus procedure first introduced by Pérez-Castrillo and Wettstein (2001). The mechanism proposed by Hart and Mas-Colell (1996) allows one active agent, randomly chosen (with uniform probability), to make a feasible proposal to all the active agents. ${ }^{4}$ This proposal has to be accepted or rejected by the rest of active agents, following some prefixed order. If they all accept the set of active agents cooperate together and distribute the value as proposed. If one agent rejects, the proposer is eliminated from the set of active agents with a certain probability and the mechanism starts all over again. The Shapley value for the grand coalition is obtained as the probability of being eliminated approaches 0 . The bidding-for-the-surplus mechanism (Pérez-Castrillo and Wettstein, 2001) differs in two aspects. First, a bidding stage is introduced, so that the proposer is not chosen randomly. Each active player chooses a bid for each of the other active players and the one with the highest net bid becomes the proposer. Second, the proposer leaves the game with probability one in case of a rejection. While Hart and Mas-Colell (1996) implement the Shapley value for any monotonic TU game, the bidding-for-the-surplus does so for zero-monotonic TU games, properties that imply in particular that the Shapley value is larger for an agent when he or she participates than when the agent is left alone. Intuitively, the advantages of being a proposer vanishes in Hart and Mas-Colell (1996) due to the fact that (i) the proposer never leaves the game, and (ii) any active agent becomes the new proposer with equal probability and the game starts all over again. In the bidding-for-the-surplus mechanism the bidding stage alleviates the advantage of being the proposer, and so does our mechanism. The proposer, at the proposal stage, will make all respondants indifferent between rejecting or not. Therefore, all active agents except the proposer obtain the payoff at the reduced game without the proposer. Hence, no rejections take place. In order to make payoffs more balanced, as in the Shapley value, the proposer always pays the value of his bid to each of the other players previous to the proposal stage. This bid coincides with the difference in payoffs to each of the other agents between the scenario with the proposer and the scenario without the proposer. The only thing that has to be satisfied is that any agent becoming the proposer prefers participating in the procedure than not to participate. That is the role played by the assumption of monotonicity or of zero-monotonicity.

Mutuswami, Pérez-Castrillo and Wettstein (2004) extend the bidding-for-the-surplus mechanism to the provision of public goods and network formation. In the latter context, the main difference with respect to the original bidding-for-the-surplus mechanism is that at the second

\footnotetext{
${ }^{4}$ Active agent means that this agent is still allowed to play.
} 
stage the proposer announces in addition a coalition and a connected graph on such coalition. Vidal-Puga and Bergantiños (2003) apply the bidding-for-the-surplus mechanism to implement the Owen value, which is an extension of the Shapley value to cooperative situations where players are organized in a-priori unions. Slikker (2007) has shown that a modification of the bidding-for-the-surplus obtains the Myerson value for monotonic value functions. As it is a procedure based on the bidding-for-the-surplus, it mainly differs from our procedure in three points. First, we propose a procedure that is based on sequential and bilateral bargaining, while the bidding-for-the-surplus is not. Second, in the bidding-for-the-surplus prices are always paid in equilibrium, whereas in our mechanism the announced prices are paid only if a proposal is rejected. In particular, no prices are paid in the unique subgame perfect equilibrium outcome. Finally, in case of a rejection, the bidding-for-the-surplus mechanism deletes all the links of the proposer, while our procedure only eliminates the link for which the players have failed to reach an agreement.

The paper is organized as follows. In Section 2 we lay out the model of a cooperative game with network structure, introduce the Myerson value, and define an illustrate the assumption of link-convexity. In Section 3 we describe the bargaining procedure and illustrate it by means of a three-player example. In Section 4 we present and prove the main result, stating that the bargaining procedure uniquely leads to the Myerson value, provided link-convexity is satisfied. The appendix contains a proof of the lemma stating that in link-convex game, the Myerson value payoff of a player in a connected component decreases if we delete a link from this component.

\section{The Model}

\subsection{Cooperation in Graphs and Value Functions}

Let $N=\{1, \ldots, n\}$ be the set of players. The bilateral negotiation possibilities among players are given by an undirected graph $g$ with $N$ being the set of nodes. Such a graph $g$ consists of a set of undirected links $(i, j)$, and the interpretation is that players $i$ and $j$ can negotiate directly if and only if $(i, j) \in g$. The coalitions in $N$ which will eventually cooperate are the connected components of $N$ in $g$. Every connected component $S$ in $g$ has a value $w(S, g)$, which is the total surplus from cooperation for $S$ if the cooperation structure is given by $g$. This value is assumed to be perfectly transferable among players in $S$.

Let $G$ be the set of all possible undirected graphs on $N$. For every graph $g$ and coalition $S$ we define the restriction of $g$ to $S$ as $g \mid S=\{(i, j) \in g: i \in S$ and $j \in S\}$. Note that $g \mid S \subseteq g$ and $g \mid N=g$. A coalition $R \subseteq S$ is called a connected component of $S$ in $g$ if: (1) for every two players in $R$, there is a path, that is, a set of consecutive links, in $g \mid S$ connecting them, and (2) for any player $i$ in $R$ and any player $j$ not in $R$, there is no path in $g \mid S$ that connects them. Let $S \mid g$ be the set of connected components of $S$ induced by $g$. Similarly, we may define $N \mid g$ as the set of connected components of $N$ in $g$. Note that $N \mid g$ is a partition of $N$. A graph $g$ is connected if $N \mid g=\{N\}$.

A value function is a map $w$ that assigns to every graph $g$ and every connected component $S$ in $g$ a value $w(S, g)$. Following Jackson and Wolinsky (1996), we assume throughout the paper that the value $w(S, g)$ does not depend on the cooperation structure outside $S$. That is, $w(S, g)=w\left(S, g^{\prime}\right)$ whenever $g\left|S=g^{\prime}\right| S$. However, we allow $w(S, g)$ to depend on the 
cooperation structure inside $S$, hence, $w(S, g)$ may differ from $w\left(S, g^{\prime}\right)$ if $g \mid S$ and $g^{\prime} \mid S$ are different. From now on, the value function $w$ is assumed to be fixed.

\subsection{The Myerson Value}

An allocation rule is a function $y$ that assigns to every graph $g \in G$ some payoff vector $y(g) \in \Re^{n}$. An allocation rule is called component efficient if for every graph $g \in G$ and every connected component $S \in N \mid g$, we have

$$
\sum_{i \in S} y_{i}(g)=w(S, g) .
$$

Let $g \backslash(i, j)$ be the graph that results after deleting the link $(i, j)$ from $g$. An allocation rule $y$ is called fair (Myerson (1977)) if for every graph $g \in G$ and every link $(i, j) \in g$, it holds

$$
y_{i}(g)-y_{i}(g \backslash(i, j))=y_{j}(g)-y_{j}(g \backslash(i, j)) .
$$

By fairness, we thus impose that two players who cooperate directly gain or lose the same amount by dissolving this cooperation.

Jackson and Wolinsky (1996) show that there is a unique component efficient and fair allocation rule, which can be written as the Shapley value of some auxiliary TU-game to be described below. This is an extension of an earlier result by Myerson (1977), who restricted attention to value functions $w$ with the property that $w(S, g)$ is independent of the cooperation structure inside and outside $S$.

For every graph $g \in G$ let $\left[N, U_{g}\right]$ be the auxiliary TU-game in which the characteristic function $U_{g}$ assigns to every coalition $S$ the value

$$
U_{g}(S)=\sum_{R \in S \mid g} w(R, g \mid S) .
$$

Intuitively, $U_{g}(S)$ is the total surplus from cooperation that players in $S$ may obtain if the cooperation structure is restricted to $g$ and no cooperation with players outside $S$ is possible. Note that if $S$ is a connected component in $g$ then $U_{g}(S)=w(S, g)$.

Theorem 2.1 (Myerson (1977) and Jackson \& Wolinsky (1996)). There is a unique component efficient and fair allocation rule, namely the allocation rule assigning to every graph $g$ the Shapley value of $\left[N, U_{g}\right]$.

Throughout the paper, the fair and component efficient allocation rule will be referred to as the Myerson value, and will be denoted by $m$. More precisely, by $m_{i}(g)$ we denote the payoff for player $i$ if the Myerson value is applied to graph $g$.

\subsection{Link-Convexity}

For the remainder of this paper we shall restrict our attention to a particular class of value functions, namely those satisfying link-convexity. In words, link-convexity states that the marginal contribution of a link to a connected component is increasing in the number of links inside this component. Formally, we have the following definition. 
Definition 2.2 A value function $w$ is called link-convex if

$$
U_{g}(S)-U_{g \backslash l^{1}}(S)>U_{g \backslash l^{2}}(S)-U_{g \backslash\left\{l^{1}, l^{2}\right\}}(S)
$$

for every graph $g$, every connected component $S \in N \mid g$ and every two links $l^{1}, l^{2}$ in $g \mid S$.

Note that link convexity implies that

$$
U_{g}(S)>U_{g \backslash l}(S)
$$

for every graph $g$, connected component $S$ in $g$ and link $l \in g \mid S$. This is obtained by considering the case $l^{1}=l^{2}$ in the definition of link-convexity.

We now derive a result that proves to be important for our main theorem. One can show, namely, that for a link-convex value function, deleting a link from a connected component leads to a strictly lower Myerson value payoff for all players in that component.

Lemma 2.3 Let $w$ be a link-convex value function. Then, $m_{i}(w, g)>m_{i}(w, g \backslash l)$ for every graph $g$, connected component $S \in N \mid g$, player $i \in S$ and link $l \in g \mid S$.

The proof for this result can be found in the appendix.

\subsection{Examples for Link-Convexity}

We conclude this section with some examples of value functions that satisfy link-convexity. In a context of TU games with communication structures, van den Nouweland and Borm (1991) find necessary and sufficient conditions for the resulting game to be link-convex, assuming the underlying TU game is convex.

\section{Example 1: Cooperation between Scientists}

Consider a group $N$ of scientists that work together on a project. Each scientist is involved in bilateral cooperations with other scientists, represented by a graph $g$. As every scientist has his own field of expertise, every bilateral cooperation, or link, yields a type of knowledge that can only be generated by this specific cooperation. For every link $l$, let $c(l)$ be a positive number that reflects the contribution of the bilateral cooperation $l$ to the project. For a connected component $S \in N \mid g$ of scientists, define its value by

$$
w(S, g)=\prod_{l \in g \mid S} c(l),
$$

whenever $S$ contains at least two members. If the connected component $S$ contains only one scientist $i$, that is, if $i$ is disconnected, then let $w(\{i\}, g)=0$. Hence, the total scientific output of the group $S$ corresponds to a Cobb-Douglas production function in the input variables $c(l)$ generated by the various bilateral cooperations inside $S$. It may be verified by the reader that such value functions $w$ are link-convex if $c(l)>2$ for all links $l$.

\section{Example 2: R\&D Networks}

Consider a group $N$ of firms producing a homogeneous good in a perfectly competitive market. Each firm may, or may not, be engaged in bilateral R\&D agreements with other firms. We 
represent these agreements by links in a graph $g$. The goal of these agreements is to reduce production costs by developing new, more efficient technologies. It is assumed that a technology resulting from an $\mathrm{R} \& \mathrm{D}$ cooperation between firms $i$ and $j$ becomes available to all firms that are directly or indirectly connected to these two firms in the graph $g$. We model this as follows. Let $C_{i}\left(x_{i}, g\right)$ be the total cost for firm $i$ of producing quantity $x_{i}$ when the collection of R\&D agreements is equal to $g$. For simplicity, we assume that, for every graph $g$, connected component $S$ in $g$, firm $i$ in $S$, link $l \in g \mid S$, and quantity $x_{i}$, the ratio

$$
\frac{C_{i}\left(x_{i}, g \backslash l\right)}{C_{i}\left(x_{i}, g\right)}
$$

is independent of the graph $g$, the firm $i$ and the quantity $x_{i}$. In words, the ratio above reflects the cost reduction induced by the $\mathrm{R} \& \mathrm{D}$ cooperation $l$. As this cost reduction is independent of $g, i$ and $x_{i}$, we may define

$$
\alpha(l)=\frac{C_{i}\left(x_{i}, g \backslash l\right)}{C_{i}\left(x_{i}, g\right)}
$$

for every link $l$. Moreover, we assume $C_{i}\left(x_{i}, g\right)=x_{i}^{2}$ for every disconnected firm $i$ in $g$. As such, the cost function for a firm $i$ in a connected component $S$ is given by

$$
C_{i}\left(x_{i}, g\right)=\frac{x_{i}^{2}}{\prod_{l \in g \mid S} \alpha(l)} .
$$

Let the market price of the good be normalized to 1. Each firm $i$ in a connected component $S$ is assumed to maximize profits given the price of the good and its cost function induced by the network $g$. This yields an optimal production level equal to

$$
x_{i}(g)=\frac{1}{2} \prod_{l \in g \mid S} \alpha(l),
$$

and a profit given by

$$
\pi_{i}(g)=\frac{1}{4} \prod_{l \in g \mid S} \alpha(l) .
$$

The value $w(S, g)$ of a connected component $S$ in an R\&D network $g$ is simply the sum of the profits for all firms in the component $S$. Hence,

$$
w(S, g)=\frac{|S|}{4} \prod_{l \in g \mid S} \alpha(l),
$$

when $S$ contains at least two firms, and $w(\{i\}, g)=\frac{1}{4}$ when $i$ is disconnected. Again, it may be verified by the reader that this value function is link-convex if $\alpha(l)>2$ for all links $l$.

\section{The Bargaining Procedure}

\subsection{Description}

We now present a simple bargaining procedure that leads to the Myerson value. Let $N$ be the set of players and $g$ a graph representing the bilateral negotiation possibilities. For every connected 
component $S$, we start the following procedure: Fix an ordering of the links in $g \mid S$ such that, whenever $(i, j)$ is not the last link in $S$, then either $i$ or $j$ (or both) are involved in some future link. For every link $(i, j)$ the negotiation between $i$ and $j$ consists of two stages:

Bidding Stage. Both players simultaneously make non-negative bids $b_{i}$ and $b_{j}$.

Proposal Stage. The player with the highest bid proposes a payoff pair $\left(x_{i}, x_{j}\right)$ which the other player can accept or reject. If the player accepts we move to the next link. If the player rejects the link $(i, j)$ is removed, the proposer pays his bid to the other player and the procedure starts all over again for the reduced graph $g \backslash(i, j)$. In case both players have chosen the same bid in the bidding stage, both will propose with equal probability.

At the end we reach a graph $g^{\prime} \subseteq g$, possibly the empty graph, where all bilateral negotiations have led to an agreement. For every connected component $S^{\prime}$ in $g^{\prime}$ with at least two players, let $X\left(S^{\prime}\right)$ be the sum of all agreed upon payoffs of all the players in $S^{\prime}$. If $X\left(S^{\prime}\right)$ does not exceed the total value $w\left(S^{\prime}, g^{\prime}\right)$, then every player $i \in S^{\prime}$ obtains, next to the bids received or paid as a result of possible previous rejections, the sum of all the agreed upon payoffs to $i$. If $X\left(S^{\prime}\right)$ exceeds the total value $w\left(S^{\prime}, g^{\prime}\right)$, no player in $S^{\prime}$ receives anything next to the bids received or paid during the negotiation process. If $S^{\prime}=\{i\}$ then player $i$ obtains, next to the bids received or paid as a result of possible previous rejections, his stand-alone value $w\left(\{i\}, g^{\prime}\right)$.

\subsection{Order of Bilateral Negotiations}

In our procedure, we have imposed a restriction on the order of the bilateral negotiations. Namely, within a connected component $S$, if $i$ and $j$ negotiate, and are not the last ones to negotiate, then either $i$ or $j$ (or both) will negotiate at least once more in the future. Put equivalently, if $i$ and $j$ are not the last ones to negotiate within $S$, then it should not be the case that both $i$ and $j$ will leave the negotiations immediately afterwards. We call such orders of bilateral negotiations regular.

The reason for this condition is that without it, there is no reason to expect a fair allocation of the surplus. Assume, namely, that within some connected component $S$ players $i$ and $j$ would negotiate, would both leave the negotiations afterwards, but some other players in $S$ would still negotiate in the future. Then, $i$ and $j$ would grab all the surplus, and leave the remaining negotiators just the minimal amount they would accept. This, of course, would never yield a fair allocation, and hence the Myerson value will surely not result if we would not impose our regularity condition on the order of bilateral negotiations.

The question remains whether we can always find a regular order of bilateral negotations. It can easily be verified that this is indeed always possible. At every stage in the procedure, namely, we can choose the next link $(i, j)$ in such a way that the set of remaining links (including $(i, j))$ is connected. If we do so, then, unless $(i, j)$ is the last link, either $i$ or $j$ will be connected to a future link, and hence either $i$ or $j$ will negotiate at least once more. So, we obtain a regular order. 


\subsection{Illustration of the Bargaining Procedure}

We illustrate the bargaining procedure by means of a three-person example. Let $g_{1}=\{(1,2),(2,3)\}$, $g_{2}=\{(1,2)\}, g_{3}=\{(2,3)\}$ and $g_{4}=\emptyset$. Let $w\left(\{1,2,3\}, g_{1}\right)=w_{123}, w\left(\{1,2\}, g_{2}\right)=w_{12}$, $w\left(\{2,3\}, g_{3}\right)=w_{23}, w\left(\{1\}, g_{3}\right)=w\left(\{1\}, g_{4}\right)=w_{1}, w\left(\{2\}, g_{4}\right)=w_{2}$ and $w\left(\{3\}, g_{2}\right)=w\left(\{3\}, g_{4}\right)=$ $w_{3}$. We use subgame perfect equilibrium to analyze the players' behavior in the bargaining procedure.

We start with the graph $g_{2}$, where only players 1 and 2 negotiate. Suppose player $i$ wins the bidding stage. Then player $j$, by rejecting player $i$ 's offer, would obtain $w_{j}+b_{i}$, where $b_{i}$ is player $i$ 's bid. Therefore, player $i$ will offer player $j$ exactly $x_{j}=w_{j}+b_{i}$. So player $i$ would propose the remaining amount $x_{i}=w_{12}-w_{j}-b_{i}$ for himself and player $j$ would accept.

Note that the payoff of the winning player is decreasing in his own bid. As a consequence, players 1 and 2 in equilibrium must choose the same bid. Namely, if $b_{i}>b_{j}$ then winner $i$ could improve his payoff by decreasing his bid a little bit. Therefore, in equilibrium, both players will be the proposer with probability one half.

Furthermore, in equilibrium the common bid $b$ must be such that both players are indifferent between being the proposer or not. Namely, if one of the players would strictly prefer being the proposer, he could increase his bid a little, become the proposer with probability one and thereby improve his payoff. If, on the other hand, the player would strictly prefer being the respondant, he could decrease his bid, become the respondant with probability one and thereby improve his payoff. We know that the payoff for player $i$ from being the proposer is equal to $x_{i}=w_{12}-w_{j}-b$ and the payoff for player $i$ from being the respondant is $w_{i}+b$. By setting the two equal we obtain that

$$
b=\frac{w_{12}-w_{1}-w_{2}}{2} .
$$

Hence, for both players $i$, the expected payoff equals the payoff from being a respondant, which, as we have seen above, is equal to

$$
w_{i}+b=\frac{w_{12}+w_{i}-w_{j}}{2} .
$$

So, the payoffs to players 1,2 and 3 in graph $g_{2}$ are given by

$$
\left(\frac{w_{12}+w_{1}-w_{2}}{2}, \frac{w_{12}+w_{2}-w_{1}}{2}, w_{3}\right)
$$

This is exactly the Myerson value for graph $g_{2}$.

Similarly, it can be checked that the bargaining procedure applied to graph $g_{3}$ leads to its corresponding Myerson value.

We finally turn to the graph $g_{1}$ and assume that the order of the links in $g_{1}$ is first $(1,2)$ and then $(2,3)$.

We start at the end of the bargaining procedure, that is, link $(2,3)$. Suppose that players 1 and 2 have agreed upon payoffs $x_{1}$ and $x_{2}$ during their negotiation. The procedure then moves to players 2 and 3. Suppose player 2 wins the bidding stage. Then player 3 , by rejecting player 2 's offer, would obtain $w_{3}+b_{2}$, where $b_{2}$ is player 2's bid. Therefore, player 2 will offer player 3 
exactly $y_{3}=w_{3}+b_{2}$. So player 2 would propose the remaining amount $y_{2}=w_{123}-x_{1}-x_{2}-w_{3}-b_{2}$ for himself. Thus, player 2's total payoff if he won the bidding stage would be

$$
x_{2}+y_{2}=w_{123}-x_{1}-w_{3}-b_{2} .
$$

On the other hand, if player 3 won the bidding stage then player 2, by rejecting 3's offer, would obtain $m_{2}\left(g_{2}\right)+b_{3}$, where $m_{2}\left(g_{2}\right)$ is the Myerson value payoff for player 2 in $g_{2}$. As shown before, if the bargaining procedure has to start all over again for graph $g_{2}$, the equilibrium payoffs will coincide with the Myerson value for $g_{2}$. If player 2 would accept player 3's offer, his total payoff would be $x_{2}+y_{2}$. So player 3 would offer player 2 exactly $y_{2}=m_{2}\left(g_{2}\right)+b_{3}-x_{2}$. Player 3 would then propose the remaining amount

$$
y_{3}=w_{123}-x_{1}-x_{2}-y_{2}=w_{123}-x_{1}-m_{2}\left(g_{2}\right)-b_{3}
$$

for himself and player 2 would accept.

Note that in both cases the total payoff of the proposer is decreasing in his own bid. By a similar argument as above in graph $g_{2}$, players 2 and 3 in equilibrium must choose the same bid. Therefore, in equilibrium, both players will be the proposer with probability one half.

Furthermore, in equilibrium the common bid $b$ must be such that both players are indifferent between being the proposer or not. The argument is the same as above in graph $g_{2}$. We know that the total payoff for player 2 from being the proposer is equal to $x_{2}+y_{2}=w_{123}-x_{1}-w_{3}-b$ and the payoff for player 2 from being the respondant is $x_{2}+y_{2}=m_{2}\left(g_{2}\right)+b$. By setting the two equal we obtain that

$$
b=\frac{w_{123}-x_{1}-w_{3}-m_{2}\left(g_{2}\right)}{2} .
$$

The reader may check that this value of $b$ also makes player 3 indifferent between being the proposer or not.

Therefore, the total payoffs for players 2 and 3, if players 1 and 2 have agreed upon $x_{1}$ and $x_{2}$, are given by

$$
x_{2}+y_{2}=m_{2}\left(g_{2}\right)+b=\frac{w_{123}-x_{1}-w_{3}+m_{2}\left(g_{2}\right)}{2},
$$

and

$$
y_{3}=w_{123}-x_{1}-m_{2}\left(g_{2}\right)-b=\frac{w_{123}-x_{1}+w_{3}-m_{2}\left(g_{2}\right)}{2} .
$$

Note that, for every $x_{1}$, we have that the difference in total payoffs between players 2 and 3 is equal to $m_{2}\left(g_{2}\right)-w_{3}=m_{2}\left(g_{1} \backslash(2,3)\right)-m_{3}\left(g_{1} \backslash(2,3)\right)$. So, for every $x_{1}$ we have fairness at the link $(2,3)$.

We now turn to link $(1,2)$. Assume first that player 1 wins the bidding stage and proposes $\left(x_{1}, x_{2}\right)$. If player 2 rejects the offer, he will obtain $m_{2}\left(g_{3}\right)+b_{1}$. If he accepts, then, by (??), his final payoff will be $\left(w_{123}-x_{1}-w_{3}+m_{2}\left(g_{2}\right)\right) / 2$. So player 1 would offer the $x_{1}$ that makes player 2 indifferent between accepting and rejecting, namely

$$
x_{1}=w_{123}-w_{3}+m_{2}\left(g_{2}\right)-2 m_{2}\left(g_{3}\right)-2 b_{1},
$$

and player 2 would accept. In particular, player 1's payoff, when being a proposer, is decreasing in his own bid. 
Assume now that player 2 wins the bidding stage and proposes $\left(x_{1}, x_{2}\right)$. Player 1 , by rejecting the offer, would obtain a payoff equal to $w_{1}+b_{2}$. On the other hand, by accepting the offer, he would obtain $x_{1}$. Player 2 will therefore propose $w_{1}+b_{2}$ to player 1 and he would accept. Player 2's total payoff, by (??), would then be

$$
x_{2}+y_{2}=\frac{w_{123}-w_{1}-w_{3}+m_{2}\left(g_{2}\right)-b_{2}}{2} .
$$

Again note that player 2's total payoff when being the proposer is decreasing in his own bid.

By a similar argument as above, we can conclude that players 1 and 2 must choose the same bid in equilibrium. So both players will be the proposer with probability one half. But then, as before, the common bid $b$ must be such that both players are indifferent between being the proposer or not. Recall that player 2, by accepting player 1's bid, obtains a total payoff of $m_{2}\left(g_{3}\right)+b$, and by being the proposer obtains a total payoff of $\left(w_{123}-w_{1}-w_{3}+m_{2}\left(g_{2}\right)-b\right) / 2$. By setting the two equal we get

$$
b=\frac{w_{123}-w_{1}-w_{3}+m_{2}\left(g_{2}\right)-2 m_{2}\left(g_{3}\right)}{3} .
$$

By combining all of the equations above, we conclude that, in equilibrium, the total payoffs are given by

$$
\begin{aligned}
x_{1} & =\frac{w_{123}+2 w_{1}-w_{3}+m_{2}\left(g_{2}\right)-2 m_{2}\left(g_{3}\right)}{3}, \\
x_{2}+y_{2} & =\frac{w_{123}-w_{1}-w_{3}+m_{2}\left(g_{2}\right)+m_{2}\left(g_{3}\right)}{3}, \\
y_{3} & =\frac{w_{123}-w_{1}+2 w_{3}-2 m_{2}\left(g_{2}\right)+m_{2}\left(g_{3}\right)}{3},
\end{aligned}
$$

which is exactly the Myerson value of $g_{1}$.

\section{The Main Result}

We now prove that the bargaining procedure always leads to the Myerson value if the value function $w$ is link-convex.

Theorem 4.1 Let $w$ be a link-convex value function and $g$ a graph. Then, there is a unique subgame perfect equilibrium outcome in the bargaining procedure, and in this outcome the total payoffs for all the players coincide with the Myerson value at $g$.

Proof. We show the statement by induction on the number of links in $g$. Assume that the graph is empty. Then, by construction of the procedure, every player receives his stand-alone value, which is also the Myerson value of the empty graph.

Now consider a graph $g$ and assume that for any subgraph $g^{\prime}$ the procedure implements the Myerson value. By the construction of the bargaining procedure it is clear that we can apply the procedure to each connected component in $g$ separately. We therefore assume, without loss of generality, that the full player set $N$ is a connected component within $g$. 
Suppose now that the procedure reaches the link $(i, j)$, that is, $i$ and $j$ must negotiate. Let $N^{\text {out }}$ be the players that will not negotiate anymore, and let $N^{\text {in }}=N \backslash N^{\text {out }}$ be those players that will negotiate at least once more, starting from link $(i, j)$. Let $X^{\text {out }}$ denote the sum of total payoffs claimed so far by the players in $N^{\text {out }}$, and let $M^{\text {out }}$ be the sum of the Myerson value payoffs for the players in $N^{\text {out }}$. We proof the following claim.

Claim. Consider the subgame that starts at link $(i, j)$, and where the players in $N^{\text {out }}$ have together claimed a total payoff of $X^{\text {out }}$. Suppose that, for every remaining link $(k, l)$, we have $X^{\text {out }}<M^{\text {out }}+\left|N^{\text {in }}\right|\left(m_{k}(g)-m_{k}(g \backslash(k, l))\right)$. Then, there is a unique subgame perfect equilibrium outcome in this subgame, where every player $k \in N^{\text {in }}$ will receive a total payoff

$$
X_{k}=m_{k}(g)+\frac{M^{\text {out }}-X^{\text {out }}}{\left|N^{\text {in }}\right|} .
$$

Proof of the Claim. We prove the claim by induction on the number of links that follow $(i, j)$. To start with, assume that $(i, j)$ is the last link. Suppose that $X^{\text {out }}<M^{\text {out }}+\left|N^{\text {in }}\right|\left(m_{i}(g)-\right.$ $\left.m_{i}(g \backslash(i, j))\right)$, that is,

$$
X^{\text {out }}<M^{\text {out }}+2\left(m_{i}(g)-m_{i}(g \backslash(i, j))\right) .
$$

Assume that players $i$ and $j$ have claimed total payoffs of $X_{i}$ and $X_{j}$ so far. If $i$ wins the bidding stage, proposes $\left(x_{i}, x_{j}\right)$, and player $j$ rejects, $j$ 's total payoff would be $m_{j}(g \backslash(i, j))+b_{i}$. Here we use the induction assumption that if the link gets broken the bargaining procedure leads to the Myerson value for $g \backslash(i, j)$. On the other hand, if player $j$ accepts, his total payoff will be $X_{j}+x_{j}$. Therefore player $i$ will choose the $x_{j}$ such that $X_{j}+x_{j}=m_{j}(g \backslash(i, j))+b_{i}$. Hence, player $j$ 's total payoff would be

$$
X_{j}+x_{j}=m_{j}(g \backslash(i, j))+b_{i},
$$

and player $i$ 's total payoff would be the remaining amount, which is

$$
\begin{aligned}
X_{i}+x_{i} & =U_{g}(N)-X^{\text {out }}-\left(X_{j}+x_{j}\right) \\
& =U_{g}(N)-X^{\text {out }}-m_{j}(g \backslash(i, j))-b_{i} .
\end{aligned}
$$

Therefore, player $i$ 's total payoff is decreasing in his own bid when he is the proposer.

Similarly, if player $j$ is the proposer, player $i$ 's total payoff would be

$$
X_{i}+x_{i}=m_{i}(g \backslash(i, j))+b_{j},
$$

and player $j$ 's total payoff would be

$$
X_{j}+x_{j}=U_{g}(N)-X^{\text {out }}-m_{i}(g \backslash(i, j))-b_{j} .
$$

Hence, also player $j$ 's total payoff is decreasing in his own bid when he is the proposer.

This implies that in equilibrium players $i$ and $j$ must choose the same bid. Namely, if $b_{i}>b_{j}$, player $i$ could improve his total payoff by lowering his bid a little and still become the proposer. Therefore players $i$ and $j$ will both become the proposer with probability one half.

Furthermore, the common bid $b$ must be such that both players are indifferent between being the proposer and the respondant. If a player would strictly prefer being the proposer, 
then he could improve his total payoff by raising his bid a little and become the proposer with probability one. If he would strictly prefer being the respondant, he could improve his total payoff by lowering his bid a little and become the respondant with probability one. It can easily be shown that such a common bid exists and is unique: If we take $b_{i}=b_{j}=b$ and set (??) equal to (??), we get the same solution for $b$ as when we set (??) equal to (??), namely

$$
b=\frac{U_{g}(N)-X^{\text {out }}-m_{i}(g \backslash(i, j))-m_{j}(g \backslash(i, j))}{2} .
$$

As $U_{g}(N)=M^{\text {out }}+m_{i}(g)+m_{j}(g)$, we have that

$$
\begin{aligned}
b & =\frac{M^{\text {out }}-X^{\text {out }}+m_{i}(g)+m_{j}(g)-m_{i}(g \backslash(i, j))-m_{j}(g \backslash(i, j))}{2} \\
& =m_{i}(g)-m_{i}(g \backslash(i, j))+\frac{M^{\text {out }}-X^{\text {out }}}{2} .
\end{aligned}
$$

Here, the second equality follows from the fact that $m_{j}(g)-m_{j}(g \backslash(i, j))=m_{i}(g)-m_{i}(g \backslash(i, j))$. Since, by assumption, $X^{\text {out }}<M^{\text {out }}+2\left(m_{i}(g)-m_{i}(g \backslash(i, j))\right)$, we obtain that $b>0$. So, the common bid is positive and therefore well-defined.

With all the above, the total expected payoffs for players $i$ and $j$ equal the total payoffs they obtain by being the respondant, namely

$$
X_{i}+x_{i}=m_{i}(g \backslash(i, j))+b \text {, and } X_{j}+x_{j}=m_{j}(g \backslash(i, j))+b .
$$

By substituting (??) into (??), and using the fact that $m_{i}(g)-m_{i}(g \backslash(i, j))=m_{j}(g)-m_{j}(g \backslash(i, j))$ we get

$$
X_{i}+x_{i}=m_{i}(g)+\frac{M^{\text {out }}-X^{\text {out }}}{2}=m_{i}(g)+\frac{M^{\text {out }}-X^{\text {out }}}{\left|N^{\text {in }}\right|},
$$

and

$$
X_{j}+x_{j}=m_{j}(g)+\frac{M^{\text {out }}-X^{\text {out }}}{2}=m_{j}(g)+\frac{M^{\text {out }}-X^{\text {out }}}{\left|N^{\text {in }}\right|} .
$$

So, the claim holds if $(i, j)$ is the last link.

Consider now a link $(i, j)$ which is not the last link, and suppose that $X^{\text {out }}<M^{\text {out }}+$ $\left|N^{i n}\right|\left(m_{k}(g)-m_{k}(g \backslash(k, l))\right)$ for every remaining link $(k, l)$. Assume also that the claim holds for all the links that follow. There are two possible cases to distinguish, namely that neither $i$ nor $j$ will not leave the negotiations after $(i, j)$, or that one of them will.

Case 1. Suppose that neither $i$ nor $j$ will leave the negotiations after $(i, j)$.

In this case, the set of inactive players $N^{\text {out }}$ will remain the same after the negotiation at $(i, j)$, and hence also the claimed amount $X^{\text {out }}$. Therefore, by our induction assumption, the eventual payoffs for the players in $N^{i n}$ are not affected at all by the negotiation at $(i, j)$, as long as the offer there is accepted. So, the only objective for players $i$ and $j$ is to make sure that the offer is accepted, and hence the claim follows rather trivially in this case.

Case 2. Suppose that player $i$ will leave the negotiations after $(i, j)$. 
Since the order of bilateral negotiations is regular, we know that player $j$ is involved in at least one other remaining link.

Assume that players $i$ and $j$ have claimed total payoffs $X_{i}$ and $X_{j}$ so far. If $i$ would win the bidding stage, propose $\left(x_{i}, x_{j}\right)$ and player $j$ rejects, then $j$ 's total payoff would be

$$
m_{j}(g \backslash(i, j))+b_{i} .
$$

If $j$ would accept, then his total payoff would be

$$
m_{j}(g)+\frac{\left(M^{\text {out }}+m_{i}(g)\right)-\left(X^{\text {out }}+X_{i}+x_{i}\right)}{\left|N^{\text {in }}\right|-1} .
$$

Here, we have used the assumption that the claim holds for the link that follows $(i, j)$. Namely, if $j$ accepts, then player $i$ would have total payoff $X_{i}+x_{i}$. Since player $i$ leaves after $(i, j)$, the total claimed amount by the inactive players after $(i, j)$ would be $X^{\text {out }}+X_{i}+x_{i}$, and the sum of the Myerson value payoffs of the inactive players after $(i, j)$ would be $\left(M^{\text {out }}+m_{i}(g)\right)$. Moreover, the number of active players after $(i, j)$ would be $\left|N^{i n}\right|-1$.

So, if $i$ proposes, then we would choose $x_{i}$ such that (??) and (??) are equal. Hence, player $i$ 's total payoff would be

$$
X_{i}+x_{i}=M^{\text {out }}+m_{i}(g)-X^{\text {out }}+\left(\left|N^{\text {in }}\right|-1\right)\left(m_{j}(g)-m_{j}(g \backslash(i, j))-b_{i}\right)
$$

and player $j$ 's total payoff would be

$$
m_{j}(g \backslash(i, j))+b_{i} .
$$

We thus see that player $i$ 's total payoff is decreasing in his own bid if he is the proposer.

If $j$ would win the bidding stage, propose $\left(x_{i}, x_{j}\right)$ and $i$ rejects, then $i$ 's total payoff would be $m_{i}(g \backslash(i, j))+b_{j}$. If $i$ would accept, then his total payoff would be $X_{i}+x_{i}$, and player $j$ 's total payoff would be

$$
m_{j}(g)+\frac{\left(M^{\text {out }}+m_{i}(g)\right)-\left(X^{\text {out }}+X_{i}+x_{i}\right)}{\left|N^{\text {in }}\right|-1} .
$$

So, player $j$ would choose $x_{i}$ such that $X_{i}+x_{i}=m_{i}(g \backslash(i, j))+b_{j}$, and hence $j$ 's own total payoff would be

$$
m_{j}(g)+\frac{\left(M^{\text {out }}+m_{i}(g)\right)-\left(X^{\text {out }}+m_{i}(g \backslash(i, j))+b_{j}\right)}{\left|N^{\text {in }}\right|-1},
$$

whereas $i$ 's total payoff would be

$$
m_{i}(g \backslash(i, j))+b_{j} .
$$

Hence, also player $j$ 's total payoff is decreasing in his own bid if he is the proposer.

Summarizing, we see that for both players the total payoff when being the proposer is decreasing in the winning bid. But then, both players must choose the same bid $b$. Namely, if $b_{i}>b_{j}$ then $i$ could improve his total payoff by lowering his bid a little and still be the proposer. Similarly when $b_{j}>b_{i}$. Hence, players $i$ and $j$ will both become the proposer with probability one half. 
Also, the common bid $b$ must be such that both players are indifferent between being the proposer and being the respondant. Namely, if a player strictly prefers being the proposer, then he could improve his total payoff by raising his bid a little and thereby become the proposer with probability one. Similarly, if the player strictly prefers being the respondant, he could do better by lowering his bid and become the respondant with probability one.

It is easily verified that such bid $b$ exists: By setting (??) equal to (??) for $b_{i}=b_{j}=b$, we obtain the same solution for $b$ as by setting (??) equal to (??), namely

$$
b=\frac{M^{\text {out }}-X^{\text {out }}+m_{i}(g)-m_{i}(g \backslash(i, j))+\left(\left|N^{\text {in }}\right|-1\right)\left(m_{j}(g)-m_{j}(g \backslash(i, j))\right)}{\left|N^{\text {in }}\right|} .
$$

As $m_{j}(g)-m_{j}(g \backslash(i, j))=m_{i}(g)-m_{i}(g \backslash(i, j))$, we obtain that

$$
b=m_{i}(g)-m_{i}(g \backslash(i, j))+\frac{M^{\text {out }}-X^{\text {out }}}{\left|N^{\text {in }}\right|},
$$

which is positive since, by assumption, $X^{\text {out }}<M^{\text {out }}+\left|N^{i n}\right|\left(m_{i}(g)-m_{i}(g \backslash(i, j))\right)$. Hence, the common bid $b$ is well-defined.

So, player $i$ 's total expected payoff is equal to his expected payoff by being the respondant, which, by (??), is equal to $m_{i}(g \backslash(i, j))+b$. By (??), we can then conclude that player $i$ 's total expected payoff is

$$
m_{i}(g)+\frac{M^{\text {out }}-X^{\text {out }}}{\left|N^{\text {in }}\right|} .
$$

Similarly, player $j$ 's total expected payoff is equal to $m_{j}(g \backslash(i, j))+b$, which, by (??) and the fact that $m_{j}(g)-m_{j}(g \backslash(i, j))=m_{i}(g)-m_{i}(g \backslash(i, j))$, is equal to

$$
m_{j}(g)+\frac{M^{\text {out }}-X^{\text {out }}}{\left|N^{\text {in }}\right|} .
$$

We now explore what the other active players after $(i, j)$ would get. Consider the subgame that starts immediately after $(i, j)$, and let $\tilde{X}^{\text {out }}, \tilde{M}^{\text {out }}$ and $\tilde{N}^{\text {in }}$ refer to this subgame. Since player $i$ leaves the negotiations after $(i, j)$, and receives total payoff $m_{i}(g)+\left(M^{\text {out }}-X^{\text {out }}\right) /\left|N^{\text {in }}\right|$, we have that

$$
\tilde{X}^{\text {out }}=X^{\text {out }}+m_{i}(g)+\frac{M^{\text {out }}-X^{\text {out }}}{\left|N^{\text {in }}\right|}=m_{i}(g)+\frac{M^{\text {out }}+\left(\left|N^{\text {in }}\right|-1\right) X^{\text {out }}}{\left|N^{\text {in }}\right|} .
$$

Obviously, $\tilde{M}^{\text {out }}=M^{\text {out }}+m_{i}(g)$ and $\left|\tilde{N}^{\text {in }}\right|=\left|N^{\text {in }}\right|-1$. As, by assumption, $X^{\text {out }}<M^{\text {out }}+$ $\left|N^{i n}\right|\left(m_{k}(g)-m_{k}(g \backslash(k, l))\right)$ for every link $(k, l)$ that comes after $(i, j)$, we may conclude that

$$
\begin{aligned}
\tilde{X}^{\text {out }} & <m_{i}(g)+M^{\text {out }}+\left(\left|N^{\text {in }}\right|-1\right)\left(m_{k}(g)-m_{k}(g \backslash(k, l))\right) \\
& =\tilde{M}^{\text {out }}+\left|\tilde{N}^{\text {in }}\right|\left(m_{k}(g)-m_{k}(g \backslash(k, l))\right)
\end{aligned}
$$

for every link $(k, l)$ that comes after $(i, j)$. But then, by our induction assumption, we may conclude that every active player $k$ after $(i, j)$ will receive total payoff

$$
m_{k}(g)+\frac{\tilde{M}^{\text {out }}-\tilde{X}^{\text {out }}}{\left|\tilde{N}^{\text {in }}\right|}=m_{k}(g)+\frac{M^{\text {out }}-X^{\text {out }}}{\left|N^{\text {in }}\right|} .
$$


Together with (??) and (??) we then obtain that every player $k \in N^{\text {in }}$ receives total payoff

$$
m_{k}(g)+\frac{M^{\text {out }}-X^{\text {out }}}{\left|N^{\text {in }}\right|},
$$

which was to show. By induction, the proof of the claim is complete.

We finally prove the statement of the theorem. Let us move to the beginning of the bargaining procedure, that is, to the first link $(i, j)$. There, obviously, $X^{\text {out }}=M^{\text {out }}=0$. Since the value function is link-convex, we know by Lemma ?? that $m_{k}(g)>m_{k}(g \backslash(k, l))$ for every link $(k, l)$. Hence, we have that $X^{\text {out }}<M^{\text {out }}+\left|N^{i n}\right|\left(m_{k}(g)-m_{k}(g \backslash(k, l))\right)$ for every link $(k, l)$. But then, by our Claim, we may conclude that there is a unique subgame perfect equilibrium outcome in the bargaining procedure, where every player $k$ receives total payoff

$$
m_{k}(g)+\frac{M^{\text {out }}-X^{\text {out }}}{\left|N^{\text {in }}\right|}=m_{k}(g) .
$$

This completes the proof of the theorem.

\section{Appendix}

Proof of Lemma ??. Let the value function $w$ be link-convex and let $g$ be a graph. Recall from Section 2.2 that the auxiliary TU-game $\left[N, U_{g}\right]$ is given by

$$
U_{g}(S)=\sum_{R \in S \mid g} w(R, g \mid S)
$$

for all coalitions $S$, and that the Myerson value at $g$ coincides with the Shapley value applied to $U_{g}$. Since $S$ is the union of connected components in $g \mid S$, we may use our terminology from Section 2.3 to write

$$
U_{g}(S)=w(S, g \mid S)
$$

We prove the following claim.

Claim. Let $g$ be a graph, $S$ a coalition, $i$ a player not in $S$, and $l$ a link in $g$ such that $S \cup\{i\}$ is a connected component in $g$, and $l \in g \mid(S \cup\{i\})$. Then,

$$
U_{g}(S \cup\{i\})-U_{g}(S)>U_{g \backslash l}(S \cup\{i\})-U_{g \backslash l}(S) .
$$

Proof of claim. By definition, we have that

$$
\begin{aligned}
& {\left[U_{g}(S \cup\{i\})-U_{g}(S)\right]-\left[U_{g \backslash l}(S \cup\{i\})-U_{g \backslash l}(S)\right] } \\
= & {[w(S \cup\{i\}, g \mid(S \cup\{i\}))-w(S, g \mid S)]-} \\
& -[w(S \cup\{i\},(g \backslash l) \mid(S \cup\{i\}))-w(S,(g \backslash l) \mid S)] \\
= & {[w(S \cup\{i\}, g \mid(S \cup\{i\}))-w(S \cup\{i\},(g \backslash l) \mid(S \cup\{i\}))] } \\
& -[w(S, g \mid S)-w(S,(g \backslash l) \mid S)] .
\end{aligned}
$$


As

$$
w(S, g \mid S)=w(S \cup\{i\}, g \mid S)-w(\{i\}, g \mid S)
$$

and

$$
\begin{aligned}
w(S,(g \backslash l) \mid S) & =w(S \cup\{i\},(g \backslash l) \mid S)-w(\{i\},(g \backslash l) \mid S) \\
& =w(S \cup\{i\},(g \backslash l) \mid S)-w(\{i\}, g \mid S)
\end{aligned}
$$

it follows that

$$
w(S, g \mid S)-w(S,(g \backslash l) \mid S))=w(S \cup\{i\}, g \mid S)-w(S \cup\{i\},(g \backslash l) \mid S) .
$$

Hence, with (??) it follows that

$$
\begin{aligned}
& {\left[U_{g}(S \cup\{i\})-U_{g}(S)\right]-\left[U_{g \backslash l}(S \cup\{i\})-U_{g \backslash l}(S)\right] } \\
= & {[w(S \cup\{i\}, g \mid(S \cup\{i\}))-w(S \cup\{i\},(g \backslash l) \mid(S \cup\{i\}))]-} \\
& -[w(S \cup\{i\}, g \mid S)-w(S \cup\{i\},(g \backslash l) \mid S)] .
\end{aligned}
$$

We distinguish two cases.

Case 1. Suppose that $l \in g \mid S$. Let $l^{1}, \ldots, l^{K}$ be the links that connect $i$ with a player in $S$. Then,

$$
\begin{aligned}
g \mid(S \cup\{i\}) & =(g \mid S) \cup\left\{l^{1}, \ldots, l^{K}\right\} \text { and } \\
(g \backslash l) \mid(S \cup\{i\}) & =((g \backslash l) \mid S) \cup\left\{l^{1}, . ., l^{K}\right\} .
\end{aligned}
$$

Hence,

$$
\begin{aligned}
& w(S \cup\{i\}, g \mid(S \cup\{i\}))-w(S \cup\{i\},(g \backslash l) \mid(S \cup\{i\})) \\
= & w\left(S \cup\{i\},(g \mid S) \cup\left\{l^{1}, \ldots, l^{K}\right\}\right)-w\left(S \cup\{i\},(g \backslash l \mid S) \cup\left\{l^{1}, \ldots, l^{K}\right\}\right) \\
> & w\left(S \cup\{i\},(g \mid S) \cup\left\{l^{1}, \ldots, l^{K-1}\right\}\right)-w\left(S \cup\{i\},(g \backslash l \mid S) \cup\left\{l^{1}, \ldots, l^{K-1}\right\}\right) \\
> & w\left(S \cup\{i\},(g \mid S) \cup\left\{l^{1}, \ldots, l^{K-2}\right\}\right)-w\left(S \cup\{i\},(g \backslash l \mid S) \cup\left\{l^{1}, \ldots, l^{K-2}\right\}\right) \\
> & \ldots \\
> & w(S \cup\{i\},(g \mid S))-w(S \cup\{i\},(g \backslash l \mid S)),
\end{aligned}
$$

where the inequalities follow from link-convexity. By (??) it follows that

$$
U_{g}(S \cup\{i\})-U_{g}(S)>U_{g \backslash l}(S \cup\{i\})-U_{g \backslash l}(S),
$$

which is the desired result. This completes the proof of Case 1.

Case 2. Suppose that $l \notin g \mid S$. Then, $g|S=(g \backslash l)| S$, which implies that

$$
w(S \cup\{i\}, g \mid S)-w(S \cup\{i\},(g \backslash l) \mid S)=0 .
$$

As, by assumption, $l \in g \mid(S \cup\{i\})$, it follows that $l=(i, j)$ for some $j \in S$. Let $l^{1}, \ldots, l^{K}$ be the links other than $l$ that connect $i$ with some player in $S$. Therefore,

$$
\begin{aligned}
g \mid(S \cup\{i\}) & =(g \mid S) \cup\left\{l, l^{1}, \ldots, l^{K}\right\} \text { and } \\
(g \backslash l) \mid(S \cup\{i\}) & =(g \mid S) \cup\left\{l^{1}, . ., l^{K}\right\} .
\end{aligned}
$$


Then,

$$
\begin{aligned}
& w(S \cup\{i\}, g \mid(S \cup\{i\}))-w(S \cup\{i\},(g \backslash l) \mid(S \cup\{i\})) \\
= & w\left(S \cup\{i\},(g \mid S) \cup\left\{l, l^{1}, \ldots, l^{K}\right\}\right)-w\left(S \cup\{i\},(g \mid S) \cup\left\{l^{1}, \ldots, l^{K}\right\}\right)>0
\end{aligned}
$$

by property (??) of link-convexity. Combining this insight with (??) and (??), we obtain that

$$
U_{g}(S \cup\{i\})-U_{g}(S)>U_{g \backslash l}(S \cup\{i\})-U_{g \backslash l}(S),
$$

which was to be shown. This completes the proof of Case 2, and hence the proof of the claim is complete.

We finally show that

$$
m_{i}(w, g)>m_{i}(w, g \backslash l)
$$

for every graph $g$, connected component $S \in N \mid g$, player $i$ in $S$ and link $l$ in $g \mid S$. Consider a graph $g$ and a connected component $S$ in $g$. Let $l$ be a link in $g \mid S$. Consider the reduced graph $g \backslash l$, and the corresponding auxiliary TU-game $\left[N, U_{g \backslash l}\right]$. Define the TU-game $\left[N, V_{g, l}\right]$, where

$$
V_{g, l}(R)=U_{g}(R)-U_{g \backslash l}(R)
$$

for all coalitions $R$. We show that for all $i \in S$ the Shapley value payoff in the game $\left[N, V_{g, l}\right]$ is strictly positive.

Let $\Phi(v)$ denote the Shapley value of the game $[N, v]$. By definition,

$$
\Phi_{i}\left(V_{g, l}\right)=\sum_{R \subseteq N \backslash\{i\}} \frac{|R| !(n-|R|-1) !}{n !}\left[V_{g, l}(R \cup\{i\})-V_{g, l}(R)\right] .
$$

Fix a player $i \in S$. We show that

$$
V_{g, l}(R \cup\{i\})-V_{g, l}(R) \geq 0
$$

for all $R \subseteq N \backslash\{i\}$, and

$$
V_{g, l}(R \cup\{i\})-V_{g, l}(R)>0
$$

for at least one such $R$. Choose an arbitrary $R \subseteq N \backslash\{i\}$. Let $C_{1}, \ldots, C_{K}$ be the connected components in $g \mid(R \cup\{i\})$, and let $C_{1}$ be the connected component that contains player $i$. We distinguish two cases.

Case 1. Suppose that $l \in g \mid C_{1}$. Then,

$$
\begin{aligned}
& V_{g, l}(R \cup\{i\})-V_{g, l}(R) \\
= & {\left[U_{g}(R \cup\{i\})-U_{g}(R)\right]-\left[U_{g \backslash l}(R \cup\{i\})-U_{g \backslash l}(R)\right] } \\
= & {[w(R \cup\{i\}, g \mid(R \cup\{i\}))-w(R, g \mid R)]-[w(R \cup\{i\},(g \backslash l) \mid(R \cup\{i\}))-w(R,(g \backslash l) \mid R)] } \\
= & {\left[w\left(C_{1}, g \mid C_{1}\right)-w\left(C_{1} \backslash\{i\}, g \mid C_{1} \backslash\{i\}\right)\right]-\left[w\left(C_{1},(g \backslash l) \mid C_{1}\right)-w\left(C_{1} \backslash\{i\},(g \backslash l) \mid C_{1} \backslash\{i\}\right)\right] } \\
= & {\left[U_{g}\left(C_{i}\right)-U_{g}\left(C_{i} \backslash\{i\}\right)\right]-\left[U_{g \backslash l}\left(C_{i}\right)-U_{g \backslash l}\left(C_{i} \backslash\{i\}\right)\right] } \\
> & 0,
\end{aligned}
$$


where the inequality follows from the claim.

Case 2. Suppose that $l \notin g \mid C_{1}$. Say, $l \in g \mid C_{k}$ with $k \neq 1$. Then,

$$
\begin{aligned}
& V_{g, l}(R \cup\{i\})-V_{g, l}(R) \\
= & {\left[U_{g}(R \cup\{i\})-U_{g}(R)\right]-\left[U_{g \backslash l}(R \cup\{i\})-U_{g \backslash l}(R)\right] } \\
= & {[w(R \cup\{i\}, g \mid(R \cup\{i\}))-w(R, g \mid R)]-[w(R \cup\{i\},(g \backslash l) \mid(R \cup\{i\}))-w(R,(g \backslash l) \mid R)] } \\
= & {\left[w\left(C_{1}, g \mid C_{1}\right)-w\left(C_{1} \backslash\{i\}, g \mid C_{1} \backslash\{i\}\right)\right]-\left[w\left(C_{1},(g \backslash l) \mid C_{1}\right)-w\left(C_{1} \backslash\{i\},(g \backslash l) \mid C_{1} \backslash\{i\}\right)\right] } \\
= & 0,
\end{aligned}
$$

since

$$
w\left(C_{1}, g \mid C_{1}\right)=w\left(C_{1},(g \backslash l) \mid C_{1}\right)
$$

and

$$
w\left(C_{1} \backslash\{i\}, g \mid C_{1} \backslash\{i\}\right)=w\left(C_{1} \backslash\{i\},(g \backslash l) \mid C_{1} \backslash\{i\}\right) .
$$

As, by assumption, $S$ is a connected component in $g, i$ is a player in $S$, and $l$ is a link in $g \mid S$, there is at least one $R \subseteq N \backslash\{i\}$ with the property that $l \in g \mid C_{1}$, namely $R=S \backslash\{i\}$. By Case 1 and Case 2 it thus follows that $V_{g, l}(R \cup\{i\})-V_{g, l}(R) \geq 0$ for all $R \subseteq N \backslash\{i\}$, and $V_{g, l}(R \cup\{i\})-V_{g, l}(R)>0$ for at least one such $R$. By (??) we may conclude that $\Phi_{i}\left(V_{g, l}\right)>0$. By linearity of the Shapley value,

$$
\Phi_{i}\left(V_{g, l}\right)=\Phi_{i}\left(U_{g}\right)-\Phi_{i}\left(U_{g \backslash l}\right)=m_{i}(w, g)-m_{i}(w, g \backslash l)>0 .
$$

This completes the proof of Lemma ??.

\section{References}

[1] Amer, R. and F. Carreras (1995), Games and Cooperation Indices. International Journal of Game Theory 24(3): 239-258.

[2] Aumann, R. J. \& R. B. Myerson (1988), Endogenous Formation of Links between Players and Coalitions: An Application of the Shapley Value, in Roth, A. (ed), "The Shapley Value", Cambridge University Press, 175-191.

[3] Bergantiños, G., F. Carreras and I. García-Jurado (1993), Cooperation when Some Players are Incompatible. Mathematical Methods of Operations Research 38(2): 187-201.

[4] Bergantiños, G., B. Casas-Méndez, and M. Vázquez-Brage (2000), A Noncooperative Bargaining Procedure Generalising the Kalai-Smorodinsky Bargaining Solution to NTU Games. International Game Theory Review 2(4): 273-286.

[5] Borm, P., G. Owen and S. Tijs (1992), On the Position Value for Communication Situations. SIAM Journal on Discrete Mathematics 5 (3): 305-320.

[6] Calvo, E., Lasaga, J. and A. van den Nouweland (1999), Values of Games with Probabilistic Graphs. Mathematical Social Sciences 37: 79-95. 
[7] Dasgupta, A. \& Y. S. Chiu (1998), On Implementation via Demand Commitment Games. International Journal of Game Theory 27 (2): 161-189.

[8] Dutta, B., A. van den Nouweland and S. Tijs (1998), Link Formation in Cooperative Situations. International Journal of Game Theory 27 (2): 245-256.

[9] De Fontenay, C.C. and J. S. Gans (2007), Bilateral Bargaining with Externalities. Mimeo. University of Melbourne.

[10] Garrat, R. \& C-Z Qin (2003a), On Cooperation Structures Resulting from Simultaneous Proposals. Economics Bulletin 3 (5): 1-9.

[11] Garrat, R. \& C-Z Qin (2003b), On Potential Maximization as a Refinement of Nash Equilibrium. Economics Bulletin 3 (12): 1-11.

[12] Hamiache, G., (1999) A Value with Incomplete Communication. Games and Economic Behavior 26(1): 59-78.

[13] Herings, P.J.J., G. van der Laan and D. Talman (2008) The Average Tree Solution for Cycle-free Graph Games. Games and Economic Behavior 62(1): 77-92.

[14] Hart, S. \& A. Mas-Colell (1996), Bargaining and Value. Econometrica 64 (2): 357-380.

[15] Jackson, M. O. \& A. Wolinsky (1996), A Strategic Model of Social and Economic Networks. Journal of Economic Theory 71: 44-74.

[16] Jackson, M. O. (2003), The Stability and Efficiency of Economic and Social Networks, in "Advances in Economic Design", S. Koray and M. Sertel (eds.) Heidelberg: SpringerVerlag, 2003. Reprinted in "Networks and Groups: Models of Strategic Formation", B. Dutta and M.O. Jackson (eds.) Heidelber:Springer-Verlag, 2003.

[17] Jackson, M. O. (2005), Allocation Rules for Network Games. Games and Economic Behavior 51(1): 128-154.

[18] Jackson, M. O. (2008), "Social and Economic Networks". Princeton University Press. Princeton and Oxford.

[19] Monderer, D. and L.S. Shapley (1996), Potential Games. Games and Economic Behavior 14(1): 124-143.

[20] Mutuswami, S., D. Pérez-Castrillo \& D. Wettstein (2004), Bidding for the Surplus: Realizing Efficient Outcomes in General Economic Environments. Games and Economic Behavior 48 (1): 111-123.

[21] Myerson, R. B. (1977), Graphs and Cooperation in Games. Mathematics of Operations Research, 2: 225-229.

[22] van den Nouweland, A. \& P. Borm (1991), On the Convexity of Communication Games. Internation Journal of Game Theory, 19 (4): 421-430. 
[23] Pérez-Castrillo, D. \& D. Wettstein (2001), Bidding for the Surplus: A Noncooperative Approach to the Shapley Value. Journal of Economic Theory, 100 (2): 274-294.

[24] Pin, P. (2008), Eight Degrees of Separation. Working Paper EUI MWP 2008/44. European University Institute.

[25] Qin, C-Z. (1996), Endogenous Formation of Cooperation Structures. Journal of Economic Theory 69 (1), 218-226.

[26] Slikker, M. (2005), Link Monotonic Allocation Schemes. International Game Theory Review 7(4): 473-489.

[27] Slikker, M. (2007), Bidding for Surplus in Network Allocation Problems. Journal of Economic Theory 137(1): 493-511.

[28] Slikker, M., B. Dutta, A. van den Nouweland \& S. Tijs (2000), Potential Maximizers and Network Formation. Mathematical Social Sciences 39 (1): 55-70.

[29] Slikker, M., R.P. Gilles, H. Norde \& S. Tijs (2005), Directed Networks, Allocation Properties and Hierarchy Formation. Mathematical Social Sciences 49 (1): 55-80.

[30] Slikker, M. \& A. van den Nouweland (2000), Network Formation Models with Costs for Establishing Links. Review of Economic Design 5 (3): 333-362.

[31] Slikker, M. \& A. van den Nouweland (2002), , Network Formation, Costs and Potential Games, in Borm, P. and H. Peters (ed.) "Chapters in Game Theory (In Honor of Stef Tijs)". Theory and Decision Library C. Springer US 2002

[32] Talman, D. \& Y. Yamamoto (2008), Average Tree Solutions and Subcore for Acyclic Graph Games. Journal of the Operations Research Society of Japan 51 (3): 187-201.

[33] Vázquez-Brage, M., I. García-Jurado \& F. Carreras (1996), The Owen value Applied to Games with Graph-restricted Communication. Games and Economic Behavior, 12: $42-53$.

[34] Vidal-Puga, J. \& G. Bergantiños (2003), An Implementation of the Owen Value. Games and Economic Behavior, 44(2): 412-427.

[35] Winter, E. (1994), The Demand Commitment Bargaining and Snowballing Cooperation. Economic Theory 4: 255-273. 